\title{
L'observation des manifestations de la compétence discursive à l'oral dans le cadre de dialogues philosophiques au secondaire
}

\author{
ÉTIENNE BOUCHARD \\ Université du Québec à Chicoutimi \\ CONSTANCE LAVOIE \\ Université du Québec à Chicoutimi \\ MATHIEU GAGNON \\ Université de Sherbrooke
}

Résumé

La dimension discursive de la communication orale en contexte scolaire demeure, à l'heure actuelle, peu appuyée sur des bases empiriques. En réponse à ce manque, nous retracerons le processus d'élaboration d'un cadre d'analyse des manifestations de la compétence discursive à l'oral dans le contexte de dialogues philosophiques. Pour ce faire, nous présenterons les résultats de la mise à l'épreuve empirique de certains indicateurs d'une grille synthèse issue de notre recension d'écrits. Enfin, nous discuterons du caractère situé de la compétence discursive, en lien avec notre contexte d'investigation.

\section{Mots-clés}

Communication orale; compétence discursive, interactions, pratique du dialogue philosophique ; grille d'analyse; didactique de l'oral

\section{Introduction}

Que ce soit en contexte scolaire ou professionnel, la communication orale est considérée par plusieurs chercheurs comme l'un des piliers de la vie sociale et de l'apprentissage (Bronckart, Bulea, \& Pouliot, 2005; Simard, Dufays, Dolz, \& GarciaDebanc, 2010). Communiquer oralement permettrait effectivement d'alimenter le processus d'apprentissage et de construire des relations sociales en passant par une dynamique d'interlocution où les locuteurs élaborent en commun un discours. En contexte scolaire, ces espaces d'interlocution (causeries, dialogues philosophiques ${ }^{1}$, groupes de discussion, conseils de coopération, etc.) supposent un lot de contraintes, d'imprévus, d'adaptations, de revirements, de changements de perspective et d'attitudes (Grandaty, 2001; Nonnon, 1996). Malgré la complexité évidente de la communication orale en interaction, les oraux polygérés demeurent les grands absents du contexte

\footnotetext{
1 «Groupe de personnes qui dialoguent ensemble autour d'une question qu'ils jugent problématique et intéressante à s'engager philosophiquement dans son exploration » (Gagnon, 2005, p. 1).
} 
scolaire. Plusieurs enseignants ont d'ailleurs toujours tendance à faire de l'exposé oral l'activité de communication principale en classe de français, langue d'enseignement (Lafontaine \& Messier, 2009), et ce, même si ce type de pratique est bien souvent monogéré et évacue, par le fait même, le cadre naturel d'interlocution de la communication orale. Cette présence marquée de l'exposé oral est sans doute en lien avec la difficulté à enseigner cet objet immatériel qu'est la communication orale. Cette intangibilité de l'oral complexifie également son évaluation en contexte scolaire (GarciaDebanc, 1999). Cela apparaît d'autant plus vrai en contexte d'interlocution où les activités discursives sont prises en charge collectivement, où la gestion des tours de parole complexifie la communication et où les interlocuteurs sont souvent appelés à coordonner plusieurs actes discursifs pour prendre part activement à l'élaboration d'un tissu discursif commun (Pekarek-Doehler, 2006b; Pochon-Berger, 2010). L'enseignement de la communication orale semble constituer une activité difficile à gérer sans indicateurs afin d'orienter le quoi enseigner et le quoi évaluer.

Puisque les élèves ont à construire un discours oral en interaction quotidiennement, il semble que l'étude des manifestations de la compétence discursive à l'oral dans le cadre d'activités scolaires interactionnelles, comme le dialogue philosophique, doit être approfondie. Dans cette optique, cet article tente de répondre à la question suivante : Quels indicateurs permettent d'observer l'élaboration et l'organisation du discours oral d'élèves durant la pratique du dialogue philosophique? D'abord, nous ciblerons et évaluerons les quelques indicateurs de la compétence discursive à l'oral qui sont actuellement utilisés au Québec et en Europe. Ensuite, nous poserons un regard critique sur différents travaux principalement issus de la linguistique afin d'en dégager un premier cadre pour l'analyse d'un discours oral en interaction en contexte scolaire. À la suite de ces analyses d'ordre théorique, nous présenterons la méthodologie utilisée pour mettre à l'épreuve empiriquement ces indicateurs dans le cadre de dialogues philosophiques au secondaire. En guise de résultats, nous présenterons de quelles manières certains indicateurs de la compétence discursive à l'oral se sont précisés à travers la mise à l'épreuve du terrain. Finalement, nous discuterons de la transversalité de notre cadre d'analyse dans d'autres activités scolaires en interaction.

\section{Problématique}

Au Québec, la grille de Préfontaine, Lebrun, et Nachbauer (1998) est utilisée dans plusieurs contextes scolaires pour observer et évaluer les quatre compétences liées à la communication orale (compétence linguistique-voix, compétence linguistique-langue, compétence discursive et la compétence communicative). Cette grille, bien qu'aidante pour l'évaluation d'oraux monogérés comme l'exposé oral, est difficilement utilisable pour les oraux polygérés comme le dialogue philosophique. De tels oraux exigent des élèves de mobiliser leur compétence discursive en interagissant avec d'autres. D'ailleurs, la grille de Préfontaine et al. (1998) ne propose que deux composantes de la compétence discursive (l'organisation du discours et sa pertinence) dont les indicateurs semblent intimement liés à des oraux monogérés. L'on peut, en effet, se questionner sur l'utilité réelle d'indicateurs comme «dosage des informations », «compréhension du sujet » et « qualité des informations » pour amener des apprenants à comprendre comment élaborer et organiser leur discours oral, a fortiori lorsque ce discours s'inscrit dans une interaction 
avec les pairs qui nécessite spontanéité, adaptation et coordination de diverses ressources langagières (Pekarek-Doehler, 2006b; Pochon-Berger, 2010). Par exemple, l'indicateur «qualité des informations» apparait nettement influencé par des intentions de communication dominantes en exposé oral, qui supposent souvent de transmettre des informations qui ont été amassées lors d'une période de préparation en fonction d'un thème bien précis. Dans ce contexte, l'indicateur «qualité des informations » peut sembler opportun, dans la mesure où il oriente l'observation vers la capacité du locuteur à sélectionner des informations pertinentes en amont de la communication orale, et ce, dans l'optique d'assurer la pertinence du discours selon le thème et l'intention de communication (décrire, expliquer, informer) ${ }^{2}$. Cependant, dans un contexte pédagogique interactif comme le dialogue philosophique, cet indicateur ne semble pas convenir, puisque l'objectif est rarement de transmettre des informations « de qualité » sur un sujet donné, mais plutôt de se positionner, de délibérer, d'écouter, de questionner, et ce, sur des thèmes rarement familiers ou déterminés à l'avance (Gagnon, 2005). Effectivement, en contexte d'interlocution, l'objectif de l'observation n'est pas de détecter des indicateurs de savoirs préalablement organisés en dehors de l'interaction orale, puisqu'il n'est pas aisé d'organiser à l'avance des interactions dont on ne connait souvent ni le sujet ni l'orientation.

À notre avis, les manières dont des élèves élaborent et organisent leur discours oral en français, langue première, demeurent encore aujourd'hui peu étudiées. Dans le passé, des recherches issues de la linguistique ont bien permis de dégager un éventail d'actes discursifs (Searle, 1972; Van Ek \& Trim, 1990) mobilisés par un locuteur (p. ex., répondre à une interrogation, exprimer un accord, réfuter une affirmation). Par contre, les travaux rattachés à l'analyse conversationnelle (Brown \& Levinson, 1987; KerbratOrecchioni, 2005), bien qu'ils apportent un éclairage essentiel sur la dimension interactionnelle de la communication orale, ne s'inscrivent que très rarement dans un contexte scolaire et, encore moins, dans un contexte scolaire de niveau secondaire où le français est la langue première des apprenants.

Pour ces raisons, les enseignants de français, langue première se retrouvent bien souvent face à des outils didactiques ambigus pour guider l'observation et l'évaluation de la compétence discursive dans des espaces interactifs à l'oral.

\section{Cadre théorique}

Dans cette section, nous définissons les concepts principaux sur lesquels s'appuie notre question de recherche, et ce, afin d'apporter un éclairage conceptuel sur le contexte d'investigation, la pratique du dialogue philosophique au secondaire, ainsi que sur l'objet investigué, la compétence discursive à l'oral.

\section{La pratique du dialogue philosophique (PDP)}

Le dialogue philosophique est une activité scolaire interactionnelle où la délibération et l'argumentation sont mises à l'avant-plan. Un dialogue philosophique typique débute avec la lecture d'une histoire philosophique près de la réalité des participants et conçue pour eux. À partir de cet élément déclencheur, les élèves sont

2 Encore faut-il que le jugement sur la qualité des informations soit appuyé sur des critères pertinents. 
invités à formuler des questions de recherche, unité pédagogique de base en PDP, puisque tout le processus est ancré dans ces questions significatives pour les élèves (Sasseville \& Gagnon, 2012). Puis, l'enseignant peut inviter les élèves à classer les questions proposées (notamment pour voir si certaines s'entrecoupent ou sont préalables à d'autres) et, à travers un processus délibératif, à en sélectionner une qui sera discutée. Ensuite, les élèves dialoguent par rapport à la question retenue. L'enseignant est vu comme un médiateur qui, à travers différentes interventions d'animation, guide les élèves dans leurs réflexions (Gagnon, 2005).

La nature philosophique de l'investigation suppose d'ancrer le dialogue dans la complexité (explorer la variété de dimensions d'un même problème), la faillibilité (considérer les idées comme falsifiables et évolutives) et la généricité (dégager les dimensions génériques d'un problème). Ces différents principes à la base du dialogue philosophique rendent probable l'émergence de points de vue variés par rapport à l'objet des échanges. Théoriquement, ces différents points de vue sont alors définis, étayés, remis en question, remaniés et parfois évacués, et ce, dans l'optique d'en arriver une compréhension plus fine, plus complexe de l'objet investigué. Cette dynamique demande aux élèves de fréquemment appuyer leurs propositions sur des raisons, des critères ou différentes illustrations, mais également de se positionner par rapport aux points de vue des interlocuteurs, ce qui les amène souvent à proposer des nuances ou à manifester des désaccords pour en arriver à un sens partagé.

\section{La compétence discursive à l'oral}

Pour définir la compétence discursive à l'oral, nous avons principalement puisé nos ancrages théoriques du côté de l'analyse du discours-en-interaction, notamment alimentée par les travaux de Vion (1992) et de Kerbrat-Orecchioni (2005). Bien qu'éloignées du contexte scolaire, ces recherches nous ont permis de dégager trois composantes de la compétence discursive qui s'inscrivent en cohérence avec les exigences des situations d'interlocution, soit les déplacements idéels, la délimitation du discours et l'organisation du discours (voir Tableau 1). Dans le cadre de cet article, nous nous concentrons sur la première composante en raison de sa forte prépondérance dans les dialogues philosophiques analysés par rapport aux deux autres composantes ${ }^{3}$.

Comme l'explique clairement Kerbrat-Orecchioni (1990, p. 17), "parler c'est échanger, et c'est changer en échangeant ». C'est probablement pour cette raison que les déplacements idéels constituent la composante de la compétence discursive à l'oral la plus importante en contexte d'interaction. Dans cette optique, les déplacements idéels renvoient à un ensemble d'actes discursifs traduisant un changement sur le plan des idées, des conceptions ou des représentations des locuteurs. Bien que nous distinguions huit indicateurs pour mettre à jour ces déplacements, nous nous concentrons sur quatre d'entre eux, soit les actes d'étayage, les actes de désaccord, les actes de reformulation et les actes de conceptualisation. Nous justifions ce choix par leur fréquence importante dans les dialogues analysés ${ }^{4}$, mais également par leur poids théorique considérable dans notre recension d'écrits.

\footnotetext{
${ }^{3}$ C'est le cas de 96,23 \% de toutes les séquences encodées par rapport à la compétence discursive à l'oral.

${ }^{4}$ Voir la partie résultats pour plus de détails.
} 
Le premier indicateur, les actes d'étayage, renvoie aux actes discursifs qui visent à accroitre la vraisemblance, la crédibilité d'une intervention précédente, dans une logique de soutien, d'appui (Apothéloz \& Miéville, 1989). Bien que certaines grilles, dont celle de Préfontaine et al. (1998), s'intéressent à des actes d'étayage spécifiques comme l'explication et l'exemplification, nous croyons que l'indicateur «actes d'étayage », en étant plus général, correspond davantage à l'analyse de discours polygérés, dans la mesure où il ne circonscrit pas le regard analytique à l'une ou l'autre des diverses relations d'étayage qui peuvent marquer le déroulement des interactions orales. Cela n'exclut pas que l'observation des actes d'étayage dans un contexte spécifique, comme le dialogue philosophique, pourrait contribuer à mettre en lumière différentes déclinaisons de cet indicateur propres à cet espace particulier.

La négociation de sens qui caractérise, à divers degrés, toute interaction orale suppose bien sûr que les idées émises soient étayées, mais également que des points de vue divergents se rencontrent et que des désaccords se manifestent (Angouri \& Locher, 2012). Il n'y a qu'à penser aux échanges observables dans certains débats scolaires qui supposent souvent de nombreux revirements et qui ne se résument pas à une succession d'arguments préalablement organisés. Dans cette optique, nous croyons, comme d'autres (Kerbrat-Orecchioni, 1990; Kim, 2001), que l'indicateur «actes de désaccord» est nécessaire pour rendre compte des situations de confrontation discursive qui marquent la plupart des oraux polygérés. Ces situations peuvent prendre la forme d'une opposition, d'une divergence ou, plus délicatement, d'une nuance. Pour Kim (2001), les actes de désaccord se définissent comme une réaction oppositionnelle explicite vis-à-vis d'une intervention précédente. Par exemple, dans le cadre de certains cercles de lecture, il est possible de voir des élèves désapprouver une interprétation formulée par un pair et proposer une interprétation différente (Hébert, 2004). Sur la base de la définition de Kim (2001), cette expression d'une idée opposée par rapport à une proposition antérieure serait de l'ordre des actes de désaccord. Dans le cadre du dialogue philosophique, il nous semble que l'observation des actes de désaccord est susceptible de nous permettre d'approfondir notre compréhension de la dynamique de déplacements idéels, dans la mesure où, à travers la manifestation d'un désaccord, le locuteur cherche en quelque sorte à transformer les croyances et les représentations de ses interlocuteurs et alimentent ainsi ces possibles déplacements (Kim, 2001).

En plus des actes d'étayage et de désaccord, il convient de s'intéresser aux actes de reformulation pour l'observation des déplacements idéels (Conseil de l'Europe, 2000; Nonnon, 1996; Vion, 1992). Nous interprétons les actes de reformulation dans deux sens complémentaires, soit l'action de formuler de nouveau des propos antérieurs sans en modifier le contenu (reprise) et l'action de formuler en d'autres mots une intervention antérieure (Vion, 2006). Les reformulations apparaissent effectivement comme des manifestations pertinentes d'un travail de co-construction de sens. Pour plusieurs (Martinot \& Romero, 2009; Vion, 1992, 2006), la caractéristique principale des actes de reformulation, bien avant leur rôle de régulation du discours (Mohamed Hassan, 2011), serait de contribuer à construire le sens d'un discours commun. Cette construction de sens passerait principalement par une complexification des énoncés antérieurs (Martinot, 2010). Par exemple, si l'enseignant demande à un élève de clarifier son intervention précédente, ce dernier va bien entendu reprendre en partie cette intervention (qui ne 
variera pas), mais va y adjoindre de nouvelles informations afin d'en clarifier ou d'en complexifier le sens.

Ce passage d'un énoncé initial à une reformulation complexifiée peut bien sûr répondre à une fonction de régulation du discours (p. ex., assurer l'intercompréhension), mais il n'en demeure pas moins que le rôle discursif premier de la reformulation est de modifier des propos antérieurs, ce qui reflète nécessairement un déplacement sur le plan des idées. Pour ce qui est des reprises, même si l'on aurait également tendance à penser qu'elles ne jouent qu'un rôle de régulation du discours (p. ex. répéter une information pour réparer un bris de communication), Vion (2006) croit qu'elles reflètent également une forme de déplacement sur le plan des idées. En effet, un locuteur-reformulateur peut difficilement reprendre des propos antérieurs sans se positionner, explicitement ou implicitement, par rapport à ces propos. Ce positionnement par rapport à l'énoncé initial, marqué par une forme ou une autre de distanciation, va nécessairement en modifier le sens, ce qui contribue davantage à l'évolution des échanges qu'à la régulation de leur déroulement. Parmi les actes de reformulation, il conviendrait de distinguer les reformulations effectuées sur sa propre parole et les reformulations effectuées sur les paroles d'autrui, chaque type étant susceptible de remplir des fonctions différentes dans le discours (Vion, 1992).

Enfin, les déplacements idéels peuvent également se manifester dans les actes de conceptualisation des élèves (Nonnon, 1996; Sasseville \& Gagnon, 2012; Van Ek \& Trim, 1990; Vion, 1992). Dans le cas de la PDP en milieu scolaire, par exemple, la définition des notions à l'étude s'appuie principalement sur le travail de conceptualisation des élèves qui sont amenés à comparer/distinguer des notions intimement liées (comme amour et amitié), à définir, à apporter des nuances de sens, etc. En effet, la signification des mots et des concepts mis en circulation dans le cadre d'une interaction orale fait l'objet de plusieurs remaniements, d'extensions de sens, de restrictions, ne serait-ce que pour en arriver ultimement à une signification partagée entre les interlocuteurs (Nonnon, 1996). L'analyse des actes de conceptualisation est, par le fait même, primordiale dans l'observation des déplacements idéels dans un discours oral polygéré.

Ces quatre indicateurs théoriques, soit les actes d'étayage, de désaccord, de reformulation et de conceptualisation, nous ont permis de mettre sur pied un premier cadre analytique pour l'observation des déplacements idéels dans le cadre de la PDP. Nous avons mis à l'épreuve ces indicateurs avec des élèves du secondaire dans le cadre de dialogues philosophiques afin de mieux les décrire, de les préciser et d'en percevoir les limites.

\section{Méthodologie}

Cette recherche, de type descriptif/interprétatif et exploratoire (Elliott \& Timulak, 2005; Savoie-Zajc, 2011; Van der Maren, 2003), visait à décrire les manifestations de la compétence discursive à l'oral dans le cadre de dialogues philosophiques. Pour ce faire, nous avons colligé des données d'observation (enregistrements vidéo) afin de dresser un portrait détaillé de ces manifestations, ce qui renvoie à une recherche descriptive et interprétative. Dans cette section, nous retraçons notre processus d'échantillonnage et nous expliquons notre méthode de collecte de données ainsi que notre démarche d'analyse. 


\section{Participants}

Dans une logique d'échantillonnage par choix raisonné (Johnson \& Christensen, 2012), nous avons sélectionné une école secondaire de la région de Québec où le dialogue philosophique est pratiqué hebdomadairement par plusieurs groupes d'élèves. Sur une base volontaire, un enseignant pratiquant le dialogue philosophique depuis plus de 10 ans avec ses élèves a manifesté son intérêt pour la recherche. Cinq groupes-classes de $4^{\mathrm{e}}$ et de $5^{\mathrm{e}}$ secondaire, composés d'élèves âgés entre 15 et 16 ans, ont été approchés pour réaliser notre collecte de données. Le recrutement des élèves à l'intérieur de chaque groupe-classe s'est effectué selon le taux de réponse à une participation volontaire (Van der Maren, 2003). Tous les élèves des groupes sélectionnés ont accepté de participer aux dialogues philosophiques filmés (pour un total de 101 élèves). La taille de notre échantillon repose sur une logique de stabilisation, dans la mesure où nous voulions suffisamment des données d'observation à analyser pour être en mesure de stabiliser raisonnablement notre cadre d'analyse et, ainsi, dégager un portrait des manifestations de la compétence discursive à l'oral dans le cadre de la PDP au secondaire.

\section{Méthode de collecte de données}

Pour mener à bien notre collecte de données, nous nous sommes principalement appuyés sur l'observation non participante. Nous avons observé cinq dialogues philosophiques d'environ 75 minutes chacun ayant tous lieu dans la classe de l'enseignant participant. Toutes les séances étaient filmées et enregistrées afin de nous permettre de les visionner à plusieurs reprises ainsi que d'effectuer la majeure partie de l'analyse à même les séquences vidéo. Lors des séances, l'ensemble des élèves du groupe-classe (de 20 à 29 élèves) étaient répartis autour d'une table ronde au centre de la classe et tous les élèves étaient invités à participer aux échanges. Les activités se sont déroulées en conformité avec les modalités de mise en œuvre décrites plus tôt, c'est-àdire qu'elles ont été amorcées en prenant appui sur un support (citation philosophique de Theodor W. Adorno ou série d'images sur le thème de la beauté), elles se sont poursuivies par la collecte et la classification de questions de recherche formulées par les élèves et elles ont évolué à travers les échanges des élèves autour de la question de recherche sélectionnée et des sujets émergeants.

\section{Analyse des données}

Les données recueillies à l'occasion de la réalisation des cinq dialogues philosophiques filmés ont fait l'objet d'une codification à l'aide du logiciel QSR Nvivo 10. Le logiciel nous a permis de codifier directement dans les séquences vidéo afin de répondre à certaines exigences de l'analyse des interactions orales, notamment liées à la préservation de leur caractère temporel et actionnel (Mondada, 2012). Seules certaines séquences types ont été transcrites en verbatim afin de permettre la transmission des résultats. Dans ces transcriptions, chaque élève a été identifié à l'aide d'un code en fonction du dialogue philosophique concerné (séance $\mathrm{A}, \mathrm{B}, \mathrm{C}, \mathrm{D}$ ou $\mathrm{E}$ ). Puisque l'ensemble des enregistrements vidéo n'a pas été transcrit en verbatim, la codification des élèves s'appuie sur l'ordre de leur prise de parole dans les séquences transcrites. Par 
exemple, le deuxième élève à prendre la parole dans les séquences transcrites de la séance $\mathrm{B}$ a été identifié par le code E2 (B). Il est à noter que, pour chaque séance, un même code n'a été attribué qu'à un seul élève. Cependant, ce même élève peut avoir été encodé différemment dans une autre séance, car le but n'était pas d'identifier les élèves, mais bien de distinguer les interlocuteurs dans chaque séance. De plus, pour rester le plus près possible des interactions analysées, un code de transcription a été utilisé afin de marquer certaines spécificités du discours oral. Pour faciliter la compréhension des extraits, ce code a toutefois été combiné avec certaines marques de ponctuation propres à l'écrit. Nous avons analysé le corpus en fonction d'une démarche d'analyse à l'aide de catégories conceptualisantes (Paillé \& Mucchielli, 2012), en ayant en tête nos ancrages théoriques, mais tout en laissant place aux catégories émergeantes dans le but de préciser et de bonifier notre cadre analytique défini en amont.

\section{Résultats}

Il convient maintenant de présenter quelques résultats issus de l'analyse des séquences vidéo. Rappelons que, parmi les différentes composantes de la compétence discursive à l'oral, nous nous concentrons sur quatre manifestations de déplacements idéels : les actes d'étayage, les actes de désaccord, les actes de reformulation et les actes de conceptualisation. Nous avons choisi de traiter de ces quatre indicateurs en fonction de leur fréquence d'encodage dans le corpus à l'étude. Pour chaque indicateur, nous présenterons les sous-indicateurs émergeants que la mise à l'épreuve terrain a permis de dégager, et ce, afin de répondre à notre question de recherche. Chaque sous-indicateur sera, ensuite, illustré à l'aide d'extraits de dialogues philosophiques.

\section{Actes d'étayage}

Les actes d'étayage représentent les manifestations de déplacements idéels les plus fréquemment observées dans les dialogues philosophiques analysés (30\% de tous les déplacements idéels encodés). L'analyse des cinq dialogues du corpus nous a amenés à distinguer deux sous-indicateurs pour les actes d'étayage, soit les interventions raisonnées ou critériées ainsi que les interventions illustratives (voir figure 1). Nous avons repéré 194 interventions illustratives (56\%) comparativement à 153 interventions raisonnées ou critériées $(44 \%)$. Les deux sous-indicateurs ont été identifiés dans tous les dialogues philosophiques du corpus à l'étude. Précisons également que nous avons observé des locuteurs étayant leur propre position, mais également des locuteurs étayant l'intervention d'autrui. 


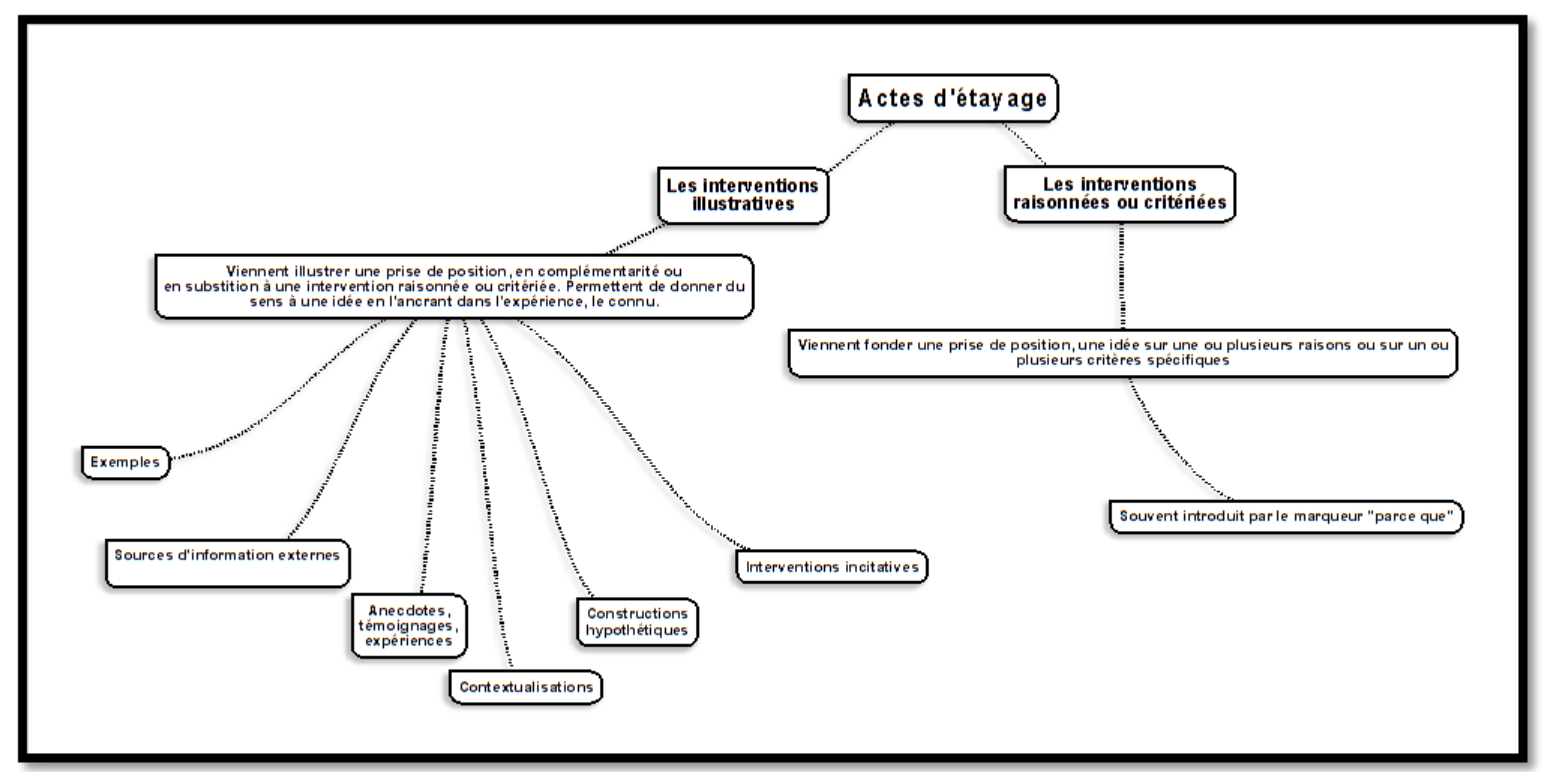

Figure 1. Schéma des actes d'étayage

Les interventions illustratives renvoient à des interventions visant, en complément ou en substitution à une intervention raisonnée ou critériée, à illustrer une prise de position ou une idée en l'ancrant dans l'expérience, dans le connu, en lui donnant une substance pour en clarifier le sens et rendre le référent intelligible pour les interlocuteurs. Ces interventions peuvent prendre une multitude de formes, comme des exemples («Dans le temps médiéval, les femmes rondes y trouvaient ça beau là »), des anecdotes ( [Pour étayer un jugement esthétique] Moi, ça me rappelle beaucoup des souvenirs- de quand j'tais jeune »), des hypothèses ( «Peut-être que eux, quand y nous r'gardent, y nous trouvent laites »), etc. Regardons une intervention illustrative où un élève incite ses pairs à imaginer, à partager une expérience en lien avec la musique. Précisons que, durant cet extrait, une musique classique jouait en arrière-plan :

É1 (A) : Avec ce genre de musique-là aussi, c'qui est fou c'est que (.) Juste le fait de fermer vos yeux ok. Pis imaginez vous de quoi qui va avec la musique pis c'qui est cool c'est qu'on aura jamais (.) la même chose qu'on va imaginer. Pis c'est ça la musique en fait, ça nous fait...

É2 (A): Rêver

L'élément d'étayage, dans ce cas-ci, constitue difficilement une raison ou un critère spécifique $^{5}$. En effet, l'É1 (A) propose plutôt aux interlocuteurs de partager une expérience afin d'ancrer dans une intelligibilité commune l'idée que la musique fait rêver chaque personne différemment. Dans cette optique, les interventions illustratives jouent un rôle complémentaire par rapport aux interventions raisonnées ou critériées.

Les interventions raisonnées ou critériées, quant à elles, comportent des éléments d'étayage spécifiques visant à fonder une prise de position antérieure. Ces éléments

${ }^{5}$ Même s'il est possible d'inférer une raison sous-jacente à l'intervention illustrative. 
d'étayage sont soit des raisons ou des critères, mais nous les avons traités sans distinction en raison de l'ambiguïté conceptuelle qui les entoure ${ }^{6}$. L'extrait suivant comporte plusieurs interventions raisonnées ou critériées proposées en rafale par rapport à la dimension esthétique d'une image représentant une femme-girafe :

Ens : Alors pourquoi vous avez dit que c'est laid ou très laid?

É3 (A) : Pa'ce que c'est pas agréable à r'garder.

É4 (A) : Ça inspire la douleur.

Ens : Chut! Pas tout l'monde en même temps. Samuel.

É5 (A) : Pa'ce que c'est différent.

La prépondérance des actes d'étayage dans les dialogues philosophiques observés nous apparaît intiment liée à la nature philosophique du processus de recherche dans lequel les élèves sont impliqués et, par extension, au caractère équivoque des concepts abordés. En effet, si les élèves interviennent aussi fréquemment pour étayer leur position, il est possible de penser que c'est en réponse à l'absence de position qui serait d'emblée considérée véridique ou définitive, de position qui ne laisserait aucunement place à la nuance ou à la contextualisation, en lien avec le principe de faillibilité sur lequel s'appuie la nature philosophique du processus de recherche (Gagnon, 2005). La nature philosophique du processus de recherche ne semble également pas indépendante de la forme qu'ont pris les actes d'étayage dans les dialogues analysés. Sur la base de nos données d'observation, il semble que l'étayage de prises de position se fasse majoritairement à travers des interventions illustratives et particulièrement à l'aide d'exemples. Cette utilisation des interventions illustratives par les élèves s'inscrit en filiation avec le principe de généricité de la PDP (Gagnon, 2005) qui suppose, d'un côté, que les problèmes de recherche soient abordés en fonction de leur dimension générique et, de l'autre, pour assurer une certaine intercompréhension, qu'il faille établir des référents communs. Il semble que les interventions illustratives, à travers les repères concrets et connus qu'elles proposent, participent à la mise en place d'un «espace minimal d'intercompréhension » (Nonnon, 1996, p. 77).

\section{Actes de reformulation}

Nous avons repéré 150 interventions traduisant un acte de reformulation dans le corpus à l'étude. Nous avons identifié autant d'actes de reformulation de la parole d'autrui que d'actes de reformulation de sa propre intervention. Pour ce qui est des actes de reformulation de la parole d'autrui, l'analyse des données nous a conduits à distinguer les marques d'adhésion à une intervention antérieure $(43,47 \%)$, les reformulations réparatrices $(39,14 \%)$ et les marques de rejet des propos d'un pair $(17,39 \%)$. Du côté

${ }^{6}$ Entre raison et concept, il semble y avoir une différence de degré, plus qu'une différence de nature, ce qui rend la distinction souvent ardue et instable. 
des actes de reformulation de sa propre intervention, nous distinguons les reformulations réparatrices $(85,58 \%)$ des reformulations de rectification $(14,42 \%)$.

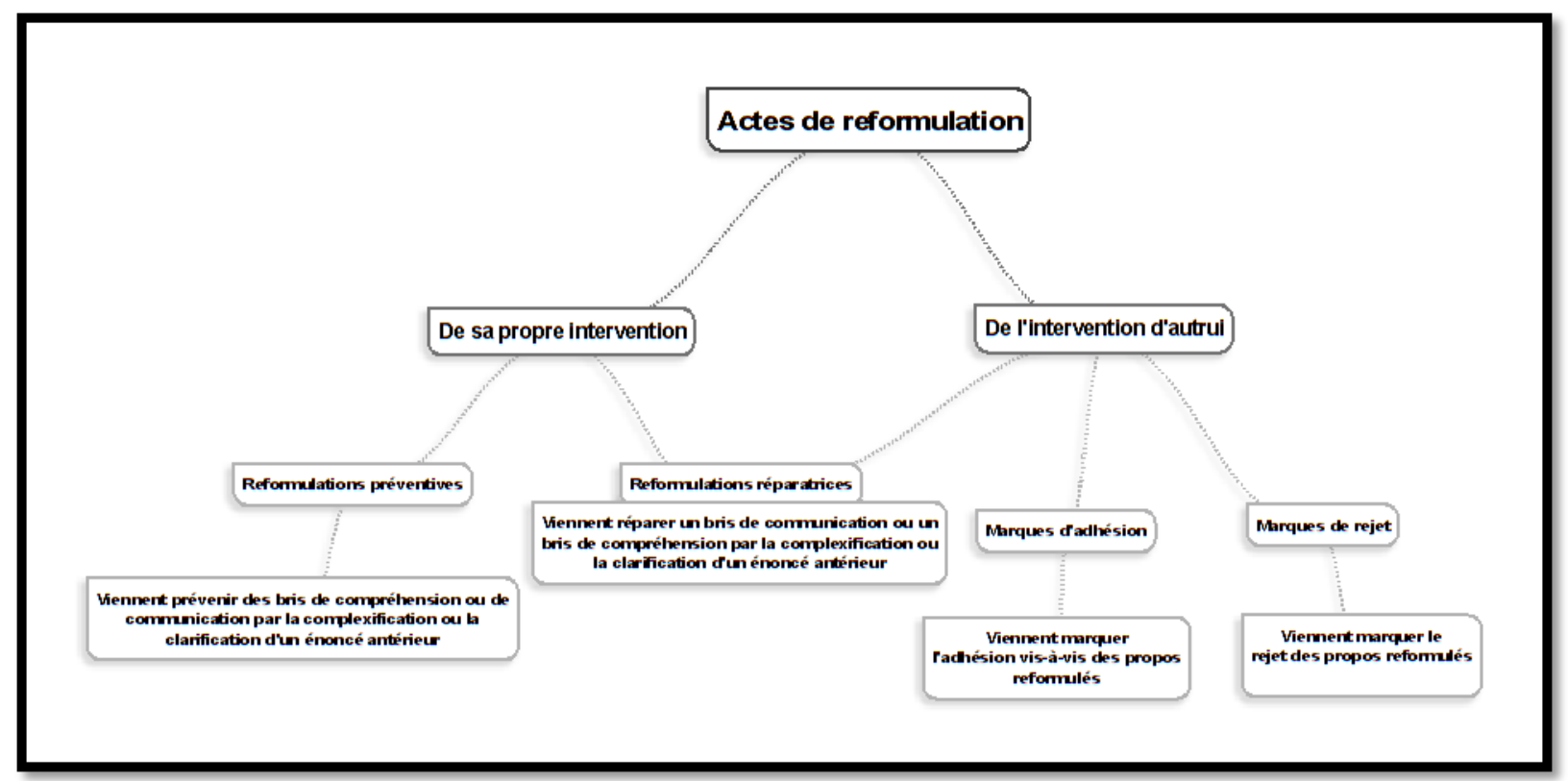

Figure 2. Schéma des actes de reformulation

Lorsque les élèves reformulent les propos d'un pair dans le cadre des dialogues philosophiques analysés, c'est principalement pour marquer leur adhésion vis-à-vis des propos reformulés, comme il est possible de l'observer dans cet échange sur la beauté d'un visage :

Ens : É2 (B) dit que c'est beau pa'ce que c'est symétrique et proportionné.

É3 (B) : ((rires)) C't'une bonne qualité.

É1 (B) : Ben ouais. Ben la normalité là.

Dans cet échange, l'intervention de É1 (B) vient marquer son adhésion aux propos de É2 (B), et ce, avec un marqueur explicite d'adhésion («Ben ouais. »). En complément à ce marqueur, É1 (B) reformule les propos d'É2 (B), ce qui semble renforcer son adhésion en signifiant que le locuteur s'associe à l'intervention précédente en la reformulant à sa manière. Pour É1 (B), ce qui est symétrique et proportionné est normal.

Que ce soit par une reformulation des paroles d'autrui ou de sa propre parole, nous avons repéré de nombreuses reformulations réparatrices (53 séquences encodées), qui venaient réparer un bris de communication (un allocutaire qui n'a pas entendu ou qui ne se rappelle plus exactement ce que le locuteur avait dit) ou un bris de compréhension (un allocutaire qui explicite ne pas comprendre une intervention du locuteur), et ce, par la complexification ou la transformation d'un énoncé antérieur. Le prochain échange, 
portant sur ce qu'il convient de croire ou de ne pas croire, contient une telle reformulation :

É6 (E) : Non mais $1+1$ (.) C'est des choses qu'on a inventées. T'sais mettons euh (.) 1+1, c'est nous qui l'a inventé fait que-c'est-c'est plus facile à croire. Mais mettons, la planète terre, tu l'as pas inventée. Comment tu pourrais croire, par exemple, qu'est ronde? J'sais pas si vous comprenez?

Ens : Non

É6 (E) : Mettons 1+1, ceux qui'ont inventé les nombres là. C'est nous qui l'a inventé. Fait qu'c'est facile de nous croire. Là tu transmets c'que t'as inventé. Mais mettons la planète ou que'que chose comme ça, c'est pas nous qui l'a inventé, fait qu'comment tu peux croire?

L'on peut observer ici que l'enseignant, qui n'a pas saisi, explicite son incompréhension à l'élève, ce qui amène ce dernier à reformuler son intervention précédente en mettant l'accent sur l'idée de transmission des inventions (ce qui constitue un déplacement par rapport à l'énoncé initial). Il s'agit d'un exemple qui reflète bien les manifestations des reformulations réparatrices, notamment puisqu'on y voit l'enseignant inciter le locuteur à une reformulation. Cette tendance est très présente dans le corpus étudié, la majorité des reformulations réparatrices étant déclenchées à la suite d'une demande de l'enseignant.

Nous avons également observé à quelques reprises un locuteur qui reformulait sa propre intervention au cours d'une même prise de parole en la complexifiant ou en la clarifiant. Le locuteur reformule une partie de son intervention, en y ajoutant des informations ou en modifiant l'élément du discours mis en relief, comme dans l'extrait qui suit où l'élève tente de déterminer des critères pour cibler quand il convient de croire en quelque chose :

Ens : J'disais comment faire pour savoir que notre jugement est bien posé pour qu'on s'trompe pas? Qu'on s'fasse pas avoir?

É2 (E) : C'est ça. Ça dépend en quoi tu veux croire ou- (.) Dans ta tête, c'est quoi qui fait que que 'que chose est vrai.

Ici, É2 (E) vient reformuler préventivement la première partie de sa réponse à la question de l'enseignant sans qu'il n'y ait eu de demande de la part des pairs ou de l'enseignant. Si dans la formulation initiale, l'élève insiste sur l'idée de "volonté de croire ", dans la reformulation, il met plutôt l'accent sur les critères pour déterminer la véracité d'une croyance. Dans cette optique, la reformulation de rectification marque un déplacement entre une idée initiale et une idée rectifiée.

En lien avec le principe de faillibilité de la PDP, la présence marquée des actes de reformulation semble traduire une disposition des élèves à revenir sur leurs points de vue, dans l'optique de les nuancer ou de les préciser en y intégrant souvent des angles 
nouveaux émergeant des interactions. En effet, il nous semble que certains actes de reformulation aient une valeur autocorrectrice, qui se traduit notamment par l'explicitation d'un glissement entre une idée initiale et une idée nouvelle (« [en parlant d'une photo de femme] Tantôt j'disais qu'était pas belle, mais avec c'que t'a dit, là j'dirais qu'elle est belle »). Bien que cette valeur autocorrectrice ne puisse être associée à tous les actes de reformulation de sa propre intervention observés, il n'est pas interdit de penser que la fréquence importante de ces actes dans le corpus étudié n'est pas indépendante de la disposition des élèves à s'autocorriger et, par extension, à la nature du processus de recherche dans la PDP (Lipman, 2003/2006). Dans une logique de pensée complexe (Morin, 2014), les reformulations, par l'orientation (adhésion c. rejet) et la complexification qu'il est possible d'y observer, s'inscrivent également dans un jeu de négociation, où les points de vue des uns se construisent sur la base des points de vue des autres, et ce, en se complexifiant toujours un peu plus.

\section{Actes de conceptualisation}

Dans notre corpus, nous avons identifié 128 séquences où les interventions des élèves traduisaient des actes de conceptualisation. Nous avons repéré davantage de moments où les actes de conceptualisation constituent la dominante de l'intervention $(75,78 \%)$ que de moments où ils ont une valeur parenthétique dans le discours $(24,22 \%)$. Les deux sous-indicateurs de l'acte de conceptualisation sont présents dans tous les dialogues philosophiques analysés. Toutefois, nous avons remarqué que les actes de conceptualisation sont plus fréquemment mobilisés dans les dialogues philosophiques portant sur des thèmes ambigus ou immatériels (la beauté, l'expertise) que dans les dialogues concernant des sujets matériels (les abattoirs, les camps de concentration).

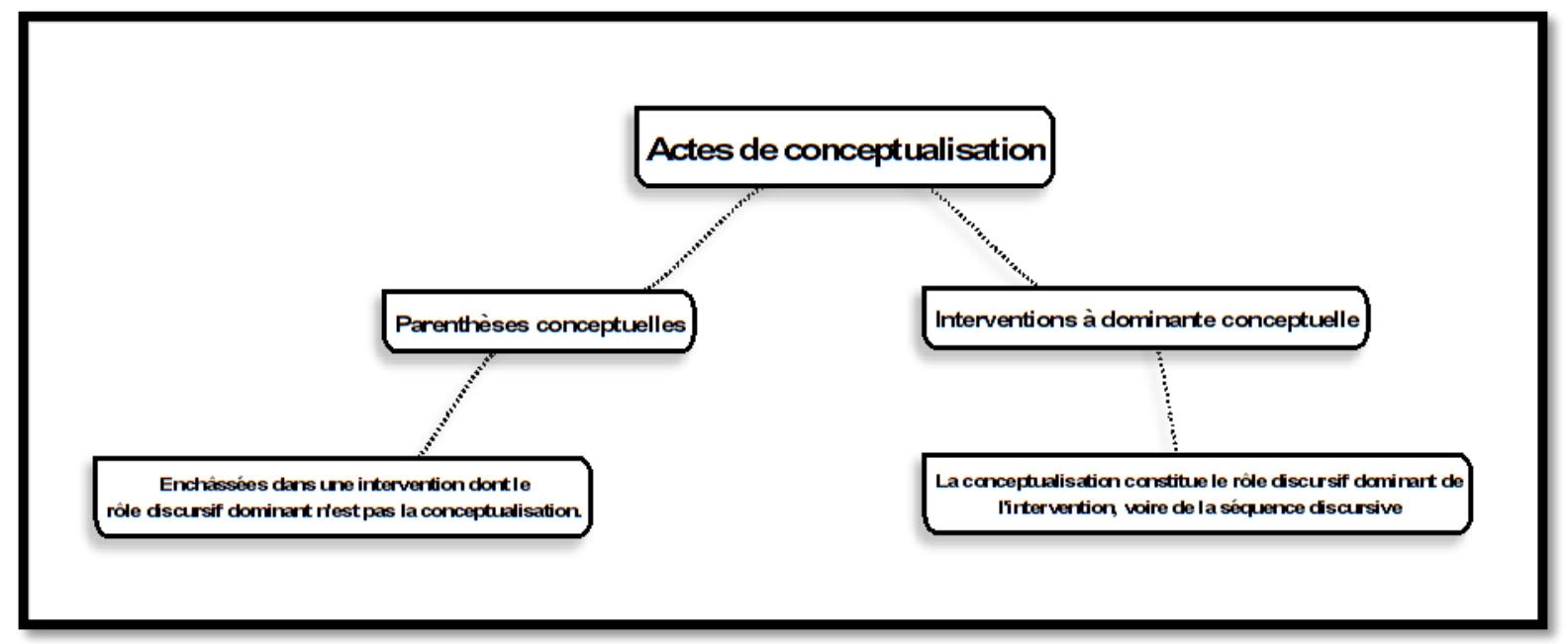

Figure 3. Schéma des actes de conceptualisation

Les interventions à visée conceptuelle renvoient à des interventions où l'acte de conceptualisation constitue la dominante discursive, c'est-à-dire que le rôle premier de l'intervention est de travailler le sens de concepts, et ce, la plupart du temps dans une séquence discursive également axée sur la conceptualisation (p. ex., une séquence où les 
élèves tentent de définir un concept comme «beauté »). L'extrait suivant présente un échange sur le thème des experts et des faux experts :

Ens : Est-ce que croire, c'est ne pas connaître?

É2 (E) : Non, croire c'est penser que que'que chose est vrai ou existe.

Dans cet extrait, l'intervention de É2 (E) vient proposer une définition de «croire» en réponse à la question de l'enseignant. Le rôle de cet acte de conceptualisation dans le discours est donc principalement de travailler le sens du concept. Les parenthèses conceptuelles, au contraire, renvoient à des actes de conceptualisation qui sont nettement enchâssés dans des interventions dont le rôle discursif n'est pas la conceptualisation, mais plutôt l'étayage ou la manifestation d'un désaccord, par exemple. La séquence qui suit illustre une parenthèse conceptuelle enchâssée dans une intervention d'étayage sur la légitimité de manger certaines viandes :

É8 (A) : On dit que mettons tu t'en vas euh :.: T'sais que dans les pays y vont manger (.) mettons le chat pis l'chien. Pour nous là, t'entends dire ça, tu fais comme- Tu trouves pas ça normal.

$[\ldots]$

É8 (A): On trouve pas ça normal. Pourquoi? Ben pa'ce qu'on a été habitué à c'que un chat pis un chien, c't'un animal de compagnie. Pis tu l'tues pas, tu l'manges pas (.)

É7 (A) : Comme y'en a qui mange pas les vaches.

Dans cette séquence, bien que É8 (A) apporte des critères définitionnels pour le concept d' " animal de compagnie », l'acte de conceptualisation est enchâssé dans une intervention à dominante d'étayage. L'intervention s'inscrit dans une série d'échanges où deux points de vue opposés par rapport à la consommation de viande (est-ce bien ou mal de manger de la viande?) sont tour à tour étayés et remis en question. L'intervention de É8 (A) vient appuyer la légitimité de manger uniquement certaines viandes, et ce, en définissant un type de viande qu'il n'est pas acceptable de manger à ses yeux.

La fréquence des actes de conceptualisation ne nous semble pas indépendante du rapport que les élèves entretiennent avec plusieurs concepts traités dans les dialogues philosophiques, des concepts considérés comme instables et abstraits (Gagnon, 2011), en lien avec le principe de généricité à la base du dispositif. La nature de ces concepts semble inciter les élèves à s'engager fréquemment dans des séquences de conceptualisation afin de poser les bases d'un réseau de significations communes. La présence des interventions à dominante conceptuelle témoigne clairement de l'importance des interventions visant à définir, à catégoriser, à comparer ou à distinguer des éléments conceptuels dans le cadre des dialogues observés. 


\section{Actes de désaccord}

Nous avons encodé un total de 119 actes de désaccord, répartis dans l'ensemble des dialogues philosophiques analysés. Nous avons majoritairement repéré des interventions de nuance $(57,98 \%)$, suivies par les interventions d'opposition $(42,02 \%)$. Il ressort donc de nos observations que lorsque les élèves explicitent un désaccord dans les dialogues philosophiques analysés, ils le font principalement pour nuancer l'intervention précédente d'un interlocuteur. Toutefois, cela ne les empêche pas de régulièrement s'opposer, de manière plus ou moins ferme, au propos d'un pair.

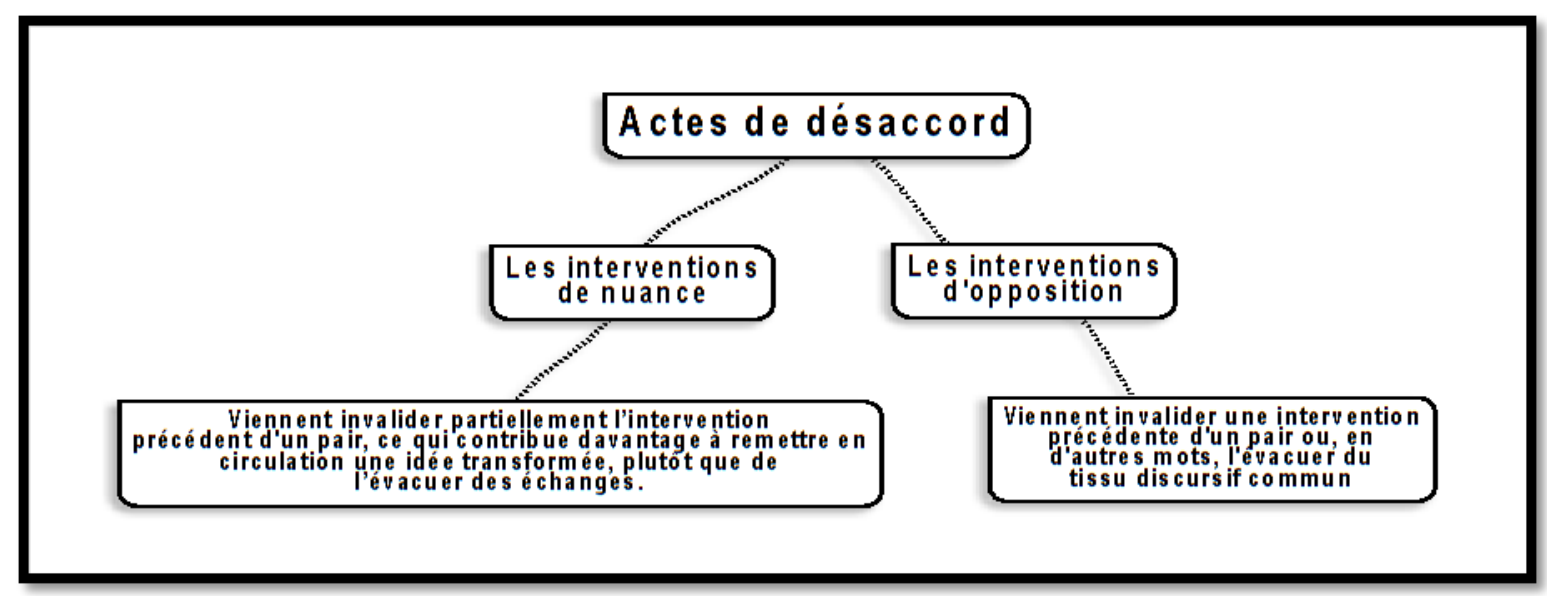

Figure 4. Schéma des actes de désaccord

Les interventions de nuance, bien qu'elles reflètent également un désaccord par rapport à une intervention précédente, ne viennent qu'invalider partiellement l'intervention d'un pair, ce qui contribue davantage à remettre en circulation une idée transformée, plutôt que de l'évacuer des échanges. Il est possible d'observer une telle intervention dans le prochain extrait où deux élèves tentent de déterminer ce qui caractérise un expert du football :

É1 (D): Non mais, mettons le football là (.) Y peut être expert pa'ce qu'i'est gros pi y'est lourd. [...]

É2 (D) : Mais le football, c'est pas jus' être gros pis rentrer dans du monde là.

É1 (D) : Non je sais, mais :.:

Dans cet exemple, É2 (D) vient nuancer l'intervention d'É1 (D) en indiquant que le football ne se résume pas aux éléments ciblés dans l'intervention précédente. Nous y observons un acte de désaccord dans la mesure où nous supposons que, si l'élève propose cette nuance, c'est probablement qu'il n'était pas en accord avec l'intervention précédente, qu'il jugeait inexacte ou limitative dans sa formulation initiale. En proposant cette nuance, É2 (D) incite donc É1 (D) à complexifier son intervention initiale. 
Contrairement aux interventions de nuance, les interventions d'opposition se traduisent par l'explicitation d'un désaccord franc qui tend nettement vers l'invalidation d'une idée ou, en d'autres mots, vers l'évacuation d'une intervention précédente du tissu discursif commun. Le prochain extrait met en scène une intervention d'opposition au cours d'une discussion sur la beauté d'un coucher de soleil :

\section{É11 (C) : Ben admettons qu'quelqu'un euh-aurait la phobie d'l'eau là, y'aurait p't'être pas marqué- qu'c'tait beau- [une image de coucher de soleil]}

\section{É2 $(C)$ : Ben là ! C'pas pa'ce que tu penses à un coucher d'soleil que tu penses que tu vas aller t'noyer là.}

Comme il est possible de le remarquer dans cette séquence, l'intervention de É2 (C) s'inscrit nettement en opposition avec l'intervention précédente de É11 (C). É2 (C) vient effectivement s'opposer au raisonnement sous-jacent à l'intervention de É11 (C), ce qui traduit un désaccord marqué. Bien que les interventions d'opposition tendent vers l'invalidation de l'intervention qu'elles ciblent, cela ne veut pas dire qu'elles sont ellesmêmes d'emblée valables.

Nous interprétons la présence marquée des actes de désaccord en lien avec la complexité qui émerge de la nature philosophique des dialogues observés. Effectivement, il nous semble que les élèves et l'enseignant se servent des positions divergentes et des opinions contrastées pour explorer en profondeur et de manière rigoureuse les sujets complexes abordés. Pour illustrer cette hypothèse, il convient d'analyser chacun des deux sous-indicateurs liés aux actes de désaccord. D’abord, du côté des interventions de nuance, elles contribuent à remettre en circulation des idées transformées, précisées. Elles semblent donc favoriser la dimension constructive et la rigueur des interactions orales en permettant de guider l'investigation en fonction d'idées jugées plus valides par les interlocuteurs ou, à tout le moins, mieux comprises par ces derniers. Du côté des interventions d'opposition, celles que nous avons observées semblent souvent tendre à évacuer des interventions jugées nettement invalides, ce qui peut également contribuer à la rigueur de la démarche de recherche philosophique sous-jacente aux interactions orales, puisque cette démarche risque moins d'être guidée par des critères, des raisonnements et des hypothèses jugés erronés par les interlocuteurs.

\section{Discussion}

Il nous semble, au terme de cette recherche, que la réponse à la question « quels indicateurs permettent d'observer l'élaboration et l'organisation du discours oral d'élèves durant la PDP? » n'est certainement pas simple, comme le prouve la diversité des indicateurs qui se sont précisés et des sous-indicateurs qui ont émergé à travers le processus d'analyse. Cette diversité mérite d'être soulignée, dans la mesure où elle traduit la complexité de la compétence discursive à l'oral, telle que mobilisée dans le cadre de dialogues philosophiques au secondaire. Dans une perspective didactique, cette complexité tend à confirmer que les balises actuellement proposées par les grilles d'observation et d'évaluation de l'oral reflètent difficilement tout ce qui est en jeu dans la 
mobilisation en interaction de la compétence discursive. Bien qu'éclairant par rapport à la manière dont les élèves du secondaire élaborent leur discours oral dans des dialogues philosophiques, le portrait que nous avons dressé ne pourrait être exporté dans une autre activité scolaire interactionnelle sans, à tout le moins, être à l'affut des particularités contextuelles qui marquent cette activité.

\section{Le caractère situé de la compétence discursive à l'oral}

Se questionner sur les indicateurs qui permettent d'observer l'élaboration du discours oral d'élèves dans un contexte pédagogique dominé par l'interaction, c'est également devoir se questionner sur le rapport qui existe entre le contexte de mobilisation de la compétence et la manière dont elle est mobilisée (Mondada, 2006). En effet, l'analyse des dialogues philosophiques nous a conduits à plusieurs reprises à réfléchir aux relations étroites qu'il est possible d'établir entre les exigences et les objectifs particuliers des dialogues philosophiques et les manifestations de déplacements idéels qu'il nous a été possible d'observer.

Comme la fréquence d'encodage des déplacements idéels en témoigne, dans le contexte des dialogues philosophiques observés, la compétence discursive à l'oral est mobilisée principalement à travers une grande variété d'actes discursifs (d'étayage, de désaccord, de reformulation, etc.) qui reflète bien la complexité de la compétence discursive et la nature tâtonnante et heuristique de la réflexion philosophique qui se déroule conjointement. En effet, il nous semble que le processus de recherche qui a pris place dans les dialogues philosophiques observés, contrairement à ce que laisse entendre Lipman (2003/2006) lorsqu'il parle du processus de recherche, ne s'est pas déployé de façon linéaire, mais bien dans une logique itérative, qui s'est traduite par de nombreux aller-retour entre la question initiale des dialogues philosophiques et les multiples ramifications explorées par les élèves. Au lieu d'une structure et d'une intention de communication prédéterminées, auxquelles l'on peut s'attendre dans un exposé oral typique et qui appellent certains actes discursifs plus que d'autres, le discours oral, dans les dialogues philosophiques observés, s'est construit progressivement et collectivement, sous l'impulsion des activités discursives et cognitives des élèves, spontanées ou sollicitées par l'enseignant, un peu à l'image d'une toile d'araignée que les locuteurs découvriraient graduellement à la lumière d'une chandelle, mais dont ils n'arriveraient pas à comprendre toute la complexité. Cette construction particulière du discours oral, liée à la nature philosophique du processus de recherche et aux interventions de l'enseignant, s'est notamment traduite par une tendance marquée chez les élèves à se situer par rapport aux sujets ambigus et équivoques des échanges, à explorer la complexité de ces sujets en explicitant les alternatives possibles, les points de vue divergents, les failles de raisonnement potentielles et à tenter de baliser les objets discursifs abordés par la conceptualisation.

Il convient alors de se demander si tous ces actes discursifs seraient mobilisés aussi fréquemment et de la même manière dans d'autres activités scolaires interactionnelles. Par exemple, pourrions-nous nous attendre à observer autant d'interventions de nuance dans un contexte de débat, où les positions des interlocuteurs sont beaucoup plus tranchées que dans un dialogue philosophique? Y aurait-il encore plus d'interventions illustratives dans le cadre d'un cercle de lecture, où l'appréciation des 
élèves doit souvent s'appuyer sur des éléments du texte à l'étude? Les actes de conceptualisation seraient-ils aussi fréquents dans des échanges en sciences, où les concepts sont vus comme beaucoup plus stables et objectifs? D'autres indicateurs et sousindicateurs pourraient-ils émerger dans d'autres contextes, selon leurs exigences et leurs objectifs particuliers? Il s'agit là de questions qui mériteraient d'être investiguées dans le cadre de recherches ultérieures.

\section{Conclusion}

En conclusion, notre recherche indique que les manifestations de la compétence discursive en contexte de dialogues philosophiques sont complexes et variées. Nous croyons que nos résultats soutiennent l'importance de préciser le regard posé sur la compétence discursive en contexte pédagogique dominé par l'interaction. Si l'on a tendance à évaluer et à enseigner cette compétence sur la base de critères larges comme la pertinence et l'organisation du discours, il convient de se demander si les enseignants gagneraient à diriger leur attention sur des actes discursifs particuliers, comme la manière dont leurs élèves étayent leur point de vue, expriment un désaccord, définissent un concept, reformulent les propos d'un pair, etc. Bref, nous nous questionnons sur la pertinence didactique de l'utilisation de critères descriptifs appuyés sur des indicateurs observables (l'élève mobilise tel acte discursif de telle manière) plutôt que de critères normatifs flous («connaissance suffisante du sujet» ou «sujet bien circonscrit»), qui peuvent faire l'objet d'interprétations fort variées, dans l'évaluation et l'enseignement de la compétence discursive à l'oral.

De plus, nous croyons que l'observation de la compétence discursive durant les oraux polygérés gagnerait à se préciser. Dans cette optique, cette recherche a permis de dégager des indicateurs et d'en décrire les manifestations, proposant de nouvelles pistes pour l'observation de la compétence discursive à l'oral et initiant du même coup une réflexion sur l'évaluation de cette compétence dans des activités scolaires interactionnelles. La manière dont nos indicateurs théoriques se sont précisés, voire transformés, par la mise à l'épreuve terrain soulignent l'importance de fournir aux enseignants des indicateurs adaptés aux différentes situations d'interlocution dans lesquelles ils sont susceptibles de placer leurs élèves, et ce, afin de soutenir adéquatement l'enseignement et l'évaluation de la compétence discursive à l'oral. Dans cette optique, réinvestir et expérimenter les indicateurs de la présente recherche dans d'autres activités scolaires interactionnelles semble représenter une avenue de recherche féconde pour alimenter la didactique de l'oral.

\section{Références}

Angouri, J., \& Locher, M. A. (2012). Theorising disagreement Journal of Pragmatics(44), 1549-1553.

Apothéloz, D., \& Miéville, D. (1989). Matériaux pour l'étude des relations argumentatives Modèles du discours. Recherches actuelles en Suisse romande. Suisse: Peter Lang. 
Bronckart, J.-P., Bulea, E., \& Pouliot, M. (2005). Introduction: pourquoi repenser l'enseignement des langues? Dans J.-P. Bronckart, E. Bulea \& M. Pouliot (Éds.), Repenser l'enseignement des langues: comment identifier et exploiter les compétences? (pp. 7-40). Lille: Presses du Septentrion.

Brown, P., \& Levinson, S. C. (1987). Politeness: Some universals in language usage. Cambridge: Cambridge University Press.

Conseil de l'Europe. (2000). Un cadre européen commun de référence pour les langues: apprendre, enseigner, évaluer. Strasbourg.

Elliott, R., \& Timulak, L. (2005). Descriptive and interpretive approaches to qualitative research. Dans J. Miles \& P. Gilbert (Éds.), A handbook of research methods for clinical and health psychology. New York Oxford University Press.

Gagnon, M. (2005). Guide pratique pour l'animation d'une communauté de recherche philosophique Québec: Les presses de l'Université Laval.

Gagnon, M. (2011). Examen des possibles relations entre la transversalité des pratiques critiques et la tranversalité des rapports aux savoirs d'adolescents du secondaire. Canadian journal of education, 34(1), 128-178.

Garcia-Debanc, C. (1999). Évaluer l'oral. Pratiques, 103/104, 193-212.

Grandaty, M. (2001). Évaluation des apprentissages oraux au cycle 1. Repères, 24-25, $137-150$.

Hébert, M. (2004). Les cercles littéraires entre pairs en première secondaire : étude des relations entre les modalités de lecture et de collaboration. Revue des sciences de l'éducation, 30(3), 605-630.

Johnson, B., \& Christensen, L. (2012). Educationnal research. Quantitative, Qualitative and Mixed Approaches (4e éd.). United States of America: SAGE Publications, Inc.

Kerbrat-Orecchioni, C. (1990). Les interactions verbales, tome 1. Paris: A. Colin.

Kerbrat-Orecchioni, C. (2005). Le discours en interaction. Paris: Armand Colin.

Kim, J.-M. (2001). Accord et désaccord dans le débat radiophonique en français et en coréen (Doctorat), Université Lumière, Lyon.

Lafontaine, L., \& Messier, G. (2009). Représentations de l'enseignement et de l'évaluation de l'oral chez des enseignants et des élèves du secondaire en français langue d'enseignement. Revue du Nouvel-Ontario, (34), 119-144.

Lipman, M. (2003/2006). À l'école de la pensée. Enseigner une pensée holistique (N. Decostre, Trad. 2e éd.). Bruxelles: de Boeck et Larcier.

Martinot, C. (2010). Reformulation et acquisition de la complexité linguistique? Travaux de linguistique, 61, 63-96.

Martinot, C., \& Romero, C. (2009). La reformulation : acquisition et diversité des discours. Cahiers de praxématique(52), 7-18.

Mohamed Hassan, R. (2011). Les croyances des enseignants et des apprenants adultes quant à la rétroaction corrective à l'oral et la pratique réelle en classe de français langue étrangère en Égypte. (Ph.D), Université de Montréal, Montréal.

Mondada, L. (2006). La compétence comme dimension située et contingente, localement évaluée par les participants. Bulletin VALS-ASLA, 84, 83-119.

Mondada, L. (2012). Video analysis and the temporality of inscriptions within social interaction: the case of architects at work. Qualitative Research, 12(3), 304-333. 
Morin, E. (2014). Introduction à la pensée complexe. Paris: Points.

Nonnon, É. (1996). Activités argumentatives et élaboration de connaissances nouvelles : le dialogue comme espace d'exploration. Langue française, 112(1), 67-87.

Paillé, P., \& Mucchielli, A. (2012). L'analyse qualitative en sciences humaines et sociales. Paris: Armand Colin.

Pekarek-Doehler, S. (2006b). Compétence et langage en action. Bulletin VALS-ASLA, 84, 4-45.

Pochon-Berger, E. (2010). La compétence d'interaction en L2: gestion de la cohérence interactive par des apprenants français. ((Thèse de doctorat inédite)), Université de Neuchâtel, Suisse.

Préfontaine, C., Lebrun, M., \& Nachbauer, M. (1998). Pour une expression orale de qualité. Montréal, Canada: Les Éditions Logiques.

Sasseville, M., \& Gagnon, M. (2012). Penser ensemble à l'école: des outils pour l'observation d'une communauté de recherche philosophique en action Québec: Les presses de l'Université Laval.

Savoie-Zajc, L. (2011). La recherche qualitative/interprétative en éducation. Dans T. Karsenti \& L. Savoie-Zajc (Éds.), La recherche en éducation. Étapes et approches (3e éd., pp. 123-148).

Searle, J. R. (1972). Les actes de langage. Essai de philosophie du langage. Paris: Hermann.

Simard, C., Dufays, J.-L., Dolz, J., \& Garcia-Debanc, C. (2010). Didactique du français langue première. Belgique: de Boeck

Van der Maren, J.-M. (2003). La recherche appliquée en pédagogie. Bruxelles: De Boeck Supérieur.

Van Ek, J. A., \& Trim, J. L. M. (1990). Threshold. Cambridge: Conseil de l'Europe.

Vion, R. (1992). La communication verbale. Analyse des interactions. Paris: Hachette Supérieur.

Vion, R. (2006). Reprises et modes d'implication énonciative. La linguistique, 42(2), 1128.

Biographies des auteurs

Étienne Bouchard termine actuellement une maîtrise en éducation à l'Université du Québec à Chicoutimi. Son projet de mémoire, financé par le CRSH et le FRQSC et dirigé par Mme Constance Lavoie et M. Mathieu Gagnon, s'intéresse aux manifestations des compétences pragmatiques dans le cadre de la pratique du dialogue philosophique au secondaire. Il est membre du Centre de recherche interuniversitaire sur la formation et la profession enseignante.

Constance Lavoie est professeure de didactique du français à l'Université du Québec à Chicoutimi. Ses travaux de recherche portent sur la didactique de l'oral au préscolaire et au primaire. Elle s'intéresse plus particulièrement à l'enseignement-apprentissage du vocabulaire et à l'intégration des patrimoines culturels oraux en classe. Elle est également chercheuse associée au CRIFPE. 


\section{Professeur agrégé à la Faculté d'éducation de l'Université de Sherbrooke, Mathieu}

Gagnon est chercheur associé au CRIFPE et chercheur régulier au GRÉÉ ainsi qu'au CRÉAS. Ses principaux travaux de recherche portent sur la pensée critique et les rapports épistémologiques aux savoirs des élèves et des enseignants, l'éducation éthique, les fondements de l'apprentissage et de l'éducation ainsi que sur la pratique du dialogue philosophique en communauté de recherche au primaire et au secondaire.

\section{Annexe 1 : Tableau 1}

\section{Tableau-synthèse des indicateurs de la compétence discursive à l'oral}

\begin{tabular}{|c|c|c|c|}
\hline $\begin{array}{l}\text { Composantes (\# de } \\
\text { séquences encodées) }\end{array}$ & Indicateurs & Définition & $\begin{array}{l}\text { Exemple de manifestations en contexte de } \\
\text { dialogues philosophiques }\end{array}$ \\
\hline \multirow[t]{8}{*}{$\begin{array}{l}\text { Déplacements idéels } \\
\text { (1147) }\end{array}$} & Actes d'étayage & $\begin{array}{l}\text { Actes discursifs qui visent à accroitre la } \\
\text { vraisemblance, la crédibilité d'une intervention } \\
\text { précédente, dans une logique de soutien, } \\
\text { d'appui (Apothéloz \& Miéville, 1989) }\end{array}$ & "J'trouve ça beau, pa'ce que c'est coloré » \\
\hline & Actes de désaccord & $\begin{array}{l}\text { Actes discursifs qui marquent l'opposition } \\
\text { (entière ou partielle) par rapport à une } \\
\text { intervention précédente (Angouri \& Locher, } \\
\text { 2012) }\end{array}$ & «C'que tu dis, c'pas prouvé rien. » \\
\hline & Actes de conceptualisation & $\begin{array}{l}\text { Actes discursifs qui visent à établir une base de } \\
\text { signification commune structurant le rapport au } \\
\text { réel des interlocuteurs (Sasseville \& Gagnon, } \\
\text { 2012) }\end{array}$ & $\begin{array}{l}\text { "[Etre expert] c'est pas nécessairement de toute } \\
\text { savoir, mais de-comme connaître parfaitement::. } \\
\text { (.) comme c'que t'as appris tu l'sais par coeur, } \\
\text { parfaitement pis genre (.) tu fais pas d'erreur dans } \\
\text { ce que tu sais. » }\end{array}$ \\
\hline & Actes de reformulation & $\begin{array}{l}\text { Actes discursifs qui se traduisent par la reprise } \\
\text { d'une intervention précédente en conservant } \\
\text { une partie invariante de l'intervention source, } \\
\text { et qui peut également intégrer une partie } \\
\text { variante (Martinot \& Romero, 2009) }\end{array}$ & $\begin{array}{l}\text { "Ça, c'est des mots qu'on a donné sur } \\
\text { que'qu'chose [...] Ouais, mais t'sais c'est jus' des } \\
\text { mots qu'on a donné sur que'qu'chose. " }\end{array}$ \\
\hline & $\begin{array}{l}\text { Actes de prise de position } \\
\text { (indicateur émergeant) }\end{array}$ & $\begin{array}{l}\text { Actes discursifs qui marquent explicitement un } \\
\text { (re)positionnement par rapport à un objet } \\
\text { discursif en circulation }\end{array}$ & «Moi, je trouve ça beau [...]» \\
\hline & Actes de questionnement & $\begin{array}{l}\text { Actes discursifs qui se traduisent par la } \\
\text { formulation d'une interrogation par rapport aux } \\
\text { objets discursifs en circulation ou, encore, une } \\
\text { intervention précédente (Van Ek \& Trim, } \\
\text { 1990) }\end{array}$ & $\begin{array}{l}\text { "Si on veut pas croire aux choses dont on } \\
\text { n'connaît rien, comment on va faire pour } \\
\text { apprendre?" }\end{array}$ \\
\hline & $\begin{array}{l}\text { Actes de rupture idéelle } \\
\text { (indicateur émergeant) }\end{array}$ & $\begin{array}{l}\text { Actes discursifs qui renvoient explicitement à } \\
\text { une coupure, une interruption dans la } \\
\text { construction d'une idée au cours de prise de } \\
\text { parole }\end{array}$ & $\begin{array}{l}\text { "Ben j'sais pas, on contrôle pas l'physique là. Ben } \\
\text { j'sais pas com-" }\end{array}$ \\
\hline & $\begin{array}{l}\text { Actes informatifs } \\
\text { (indicateur émergeant) }\end{array}$ & $\begin{array}{l}\text { Actes discursifs à travers lesquels un locuteur } \\
\text { propose simplement une information, un } \\
\text { élément de connaissance lancé dans } \\
\text { l'interaction comme un fait }\end{array}$ & «Ouais, c'est ça. C'est du Pop art. » \\
\hline $\begin{array}{l}\text { Délimitation et } \\
\text { bornage du discours } \\
(37)\end{array}$ & $\begin{array}{l}\text { Circonscription de la portée du } \\
\text { discours } \\
\text { (indicateur émergeant) }\end{array}$ & $\begin{array}{l}\text { Actes discursifs qui visent à clarifier } \\
\text { l'intention de communication, la portée des } \\
\text { propos ou, en d'autres mots, leur champ } \\
\text { d'influence }\end{array}$ & $\begin{array}{l}\text { "Non mais, j'parle pas d'une personne, j'parle } \\
\text { plus d'un- d'un fait. » }\end{array}$ \\
\hline \multirow[t]{2}{*}{$\begin{array}{l}\text { Organisation } \\
\text { discours }(8)\end{array}$} & \begin{tabular}{lr} 
Marques & \multicolumn{2}{c}{ explicites } \\
d'anticipation & (Conseil de \\
l'Europe, 2000) & \\
\end{tabular} & $\begin{array}{l}\text { Utilisation d'organisateurs explicites pour } \\
\text { marquer l'organisation du discours individuel }\end{array}$ & «Eille j'ai deux affaires à dire. Premièrement- » \\
\hline & $\begin{array}{l}\text { Brouillons de l'oral } \\
\text { (indicateur émergeant) }\end{array}$ & $\begin{array}{l}\text { Utilisation de l'écrit en cours d'interaction afin } \\
\text { d'organiser le discours oral se traduisant par } \\
\text { des manifestations non verbales }\end{array}$ & $\begin{array}{l}\text { (regard à ses notes, utilisation du crayon pour } \\
\text { marquer la progression dans les différents points à } \\
\text { aborder durant la prise de parole) }\end{array}$ \\
\hline
\end{tabular}

